\title{
Structural, functional and molecular dynamics analysis of cathepsin B gene SNPs associated with tropical calcific pancreatitis, a rare disease of tropics
}

\author{
Garima Singh ${ }^{1}$, Sri Krishna jayadev Magani ${ }^{1}$, Rinku Sharma ${ }^{1}$, Basharat Bhat ${ }^{1}$, Ashish Shrivastava ${ }^{1}$, \\ Madhusudhan Chinthakindi ${ }^{2}$, Ashutosh Singh ${ }^{\text {Corresp. } 1}$ \\ ${ }^{1}$ Department of Life Sciences, School of Natural Sciences, Shiv Nadar University, GREATER NOIDA, UTTAR PRADESH, India \\ Department of Surgical Gastroenterology, Osmania General Hospital, Hyderabad, India \\ Corresponding Author: Ashutosh Singh \\ Email address: ashutosh.singh@snu.edu.in
}

Tropical Calcific Pancreatitis (TCP) is a neglected juvenile form of chronic non-alcoholic pancreatitis. Cathepsin B (CTSB), a lysosomal protease involved in the cellular degradation process, has recently been studied as a potential candidate gene in the pathogenesis of TCP. According to Cathepsin B hypothesis, mutated CTSB can lead to premature intracellular activation of trypsinogen, a key regulatory mechanism in pancreatitis. So far, CTSB mutations have been studied in pancreatitis and neurodegenerative disorders, but little is known about the structural and functional effect of variants in CTSB. In this study, we investigated the effect of single nucleotide variants (SNVs) specifically associated with TCP, using molecular dynamics and simulation algorithms. There were two nonsynonymous variants (L26V and S53G) of CTSB, located in the propeptide region. We tried to predict the effect of these variants on structure and function using multiple algorithms: SIFT, Polyphen2, PANTHER, SDM sever, i-Mutant2.0 suite, mCSM algorithm, and Vadar. Further, using databases like miRdbSNP, PolymiRTS, and miRNASNP, two SNPs in the 3'UTR region were predicted to affect the miRNA binding sites. Structural mutated models of nsSNP mutants (L26V and S53G) were prepared by MODELLER v9.15 and evaluated using TM-Align, Verify 3D, ProSA and Ramachandran plot. The 3D mutated structures were simulated using GROMACS 5.0 to predict the impact of these SNPs on protein stability. The results from in silico analysis and molecular dynamics simulations suggested that these variants in the propeptide region of Cathepsin B could lead to structural and functional changes in the protein and thus could be pathogenic. Hence, the structural and functional analysis results have given interim conclusions that these variants can have a deleterious effect in TCP pathogenesis, either uniquely or in combination with other mutations. Thus, it could be extrapolated that Cathepsin $B$ gene can be screened in samples from all TCP patients in future, to decipher the distribution of variants in patients. 


\section{Structural, functional and molecular dynamics analysis of Cathepsin $B$ gene}

\section{SNPs associated with tropical calcific pancreatitis, a rare disease of tropics.}

3 Garima Singh ${ }^{1}$, Shri Krishna jayadev Magani $^{1}$, Rinku Sharma ${ }^{1}$, Basharat Bhat $^{1}$, Ashish

4 Shrivastava $^{1}$, Madhusudhan Chinthakindi ${ }^{2}$ and Ashutosh Singh ${ }^{1 *}$

$5{ }^{1}$ School of Natural Sciences, Department of Life Sciences, Shiv Nadar University, Greater

6 Noida, UP, India

$7 \quad 2$ Department of Surgical Gastroenterology, Osmania General Hospital, Hyderabad, India.

8 * Corresponding Authors (ashutosh.bio@gmail.com)

\section{Abstract}

10 Tropical Calcific Pancreatitis (TCP) is a neglected juvenile form of chronic non-alcoholic 11 pancreatitis. Cathepsin $B$ (CTSB), a lysosomal protease involved in the cellular degradation 12 process, has recently been studied as a potential candidate gene in the pathogenesis of TCP. 13 According to Cathepsin $B$ hypothesis, mutated CTSB can lead to premature intracellular activation of trypsinogen, a key regulatory mechanism in pancreatitis. So far, CTSB mutations

15 have been studied in pancreatitis and neurodegenerative disorders, but little is known about the structural and functional effect of variants in CTSB. In this study, we investigated the effect of

17 single nucleotide variants (SNVs) specifically associated with TCP, using molecular dynamics and simulation algorithms. There were two non-synonymous variants (L26V and S53G) of CTSB, located in the propeptide region. We tried to predict the effect of these variants on structure and function using multiple algorithms: SIFT, Polyphen2, PANTHER, SDM sever, iMutant2.0 suite, mCSM algorithm, and Vadar. Further, using databases like miRdbSNP, PolymiRTS, and miRNASNP, two SNPs in the 3'UTR region were predicted to affect the 
23 miRNA binding sites. Structural mutated models of nsSNP mutants (L26V and S53G) were

24 prepared by MODELLER v9.15 and evaluated using TM-Align, Verify 3D, ProSA and

25 Ramachandran plot. The 3D mutated structures were simulated using GROMACS 5.0 to predict

26 the impact of these SNPs on protein stability. The results from in silico analysis and molecular

27 dynamics simulations suggested that these variants in the propeptide region of Cathepsin $B$ could

28 lead to structural and functional changes in the protein and thus could be pathogenic. Hence, the

29 structural and functional analysis results have given interim conclusions that these variants can

30 have a deleterious effect in TCP pathogenesis, either uniquely or in combination with other

31 mutations. Thus, it could be extrapolated that Cathepsin B gene can be screened in samples from

32 all TCP patients in future, to decipher the distribution of variants in patients.

\section{Introduction}

Pancreatitis is a multifactorial, heterogeneous disease with enigmatic etiologies. It is an inflammatory condition leading to morphological changes in the pancreas, causing-pain and functional abnormalities. Alcohol(1), malnutrition(2), gallstones(3), familial clustering (4) and sometimes severe infections (5) have been observed to be significant causes of pancreatitis. Pancreatitis is broadly classified (6) as acute and chronic. Tropical Calcific Pancreatitis (TCP) (7) is a juvenile form of chronic calcific non-alcoholic pancreatitis. It is a form of Idiopathic Chronic Pancreatitis (ICP), mostly reported in developing tropical countries. The phenotypic heterogeneity (8) includes abdominal pain, ductal dilation, large pancreatic calculi, and pancreatic atrophy. The genetic heterogeneity related to TCP is still unexplored. Fibrocalculous pancreatic diabetes (FCPD) (9), a unique form of diabetes, is the unique secondary feature of TCP. TCP progresses gradually to FCPD and then at a later age, TCP patient suffers from pancreatic cancer (10). 
46 The pathophysiology of the pancreas is composed of an exocrine gland which is responsible for 47 digesting food and an endocrine gland critical for glucose homeostasis. Trypsinogen, cathepsins, 48 serine proteases, calcium sensing receptors are some of the essential genes for pancreatic 49 function regulation. According to the trypsin-centred theory of pancreatitis, trypsinogen, a key 50 zymogen in pancreatic juice and a key regulator of digestion, exhibits premature activation in 51 acinar cells of the pancreas during pancreatitis. This aberrant activation of trypsinogen leads to 52 activation of other zymogens in the pancreas itself, thereby resulting in inflammation and 53 autodigestion of pancreas. Although TCP is a distinct form of pancreatitis without a known 54 cause, what remains undebated is the initial step during initiation of TCP, which is the premature activation of trypsinogen in the pancreas itself. The mortality rate in TCP is as high as $17 \%$, and patients majorly die because of pancreatic cancer at a later stage (10).

57 Recently, we have built a database (11), mutTCPdb, which is a comprehensive database, giving 58 details about the genes and variants predicted to be associated with TCP until now. Activity of trypsin inside the pancreas is the primary critical factor in pathogenesis of TCP, and all the risk genes predicted to date, are known to regulate trypsin activity like chymotrypsin $\mathrm{C}(C T R C)$, cystic fibrosis transmembrane conductance regulator (CFTR), serine protease inhibitor Kazaltype I (SPINK1) and Cathepsin B (CTSB). According to "Cathepsin B hypothesis", CTSB plays an essential role in the premature activation of trypsinogen in the pancreas, primarily due to colocalization of Cathepsin $B$ and zymogens (12). The precise rationale behind this

65 colocalization is yet unknown. The reason could be aberrant trafficking mechanism of procathepsin $\mathrm{B}$, due to mutations in procathepsin $\mathrm{B}$ or deleterious mutations in the molecules

67 associated with the trafficking of procathepsin B, in a diseased state. 
68 A research article in 2006, described two missense mutations in CTSB (L26V and S53G), 69 identified in TCP patients from Asian Institute of Gastroenterology, Hyderabad (India) (13). The 70 minor allele frequency (MAF) of variants L26V and S53G in TCP patients were 0.46 and 0.09 71 respectively. Also in 2008, an article suggested that coexistence of variants in transcription factor 72 7-like 2 (TCF7L2), SPINK1 and CTSB (L26V), might lead to exocrine damage in TCP and 73 determine the onset of FCPD (14). The analysis in this paper (14) was performed with TCP 74 patients and control population from Dravidian and Indo-European ethnicities. There is another article which described a missense mutation (p. Q334P) in cathepsin B gene discovered in chronic pancreatitis patients but not in TCP patients (15). An article in 2014 illustrated no association of L26V mutation with TCP (16). In this paper, statistical significance tests have indicated the lack of association of L26V mutation with TCP, but this mutation was observed in 7 out of 150 TCP patients. Hence, the association of this mutation with TCP cannot be completely disregarded (16). Although researchers have identified SNVs in CTSB gene observed in TCP patients, lacunae lie in the information about the functional effect of these SNVs in the pathogenesis of TCP.

Human Cathepsin B (catB, E.C 3.4.22.1) is a lysosomal cysteine protease which is involved in several cellular processes like protein degradation, extracellular matrix degradation, regulatory mechanisms, cell death, autophagy and antigen representation (17). It belongs to papain superfamily and acts both as an endopeptidase and as an exopeptidase. Cathepsin $B$ is synthesized as an inactive proenzyme (cathepsin $B)$ and is activated by other proteases and by autocatalytic processes (18). Procathepsin B (length of protein $=339$ aa) has an N-terminus propeptide of 62 amino acid length from Arg-Lys (18-79 residues). Signal sequence (1-17 residues) and post-translational glycosylation modification (19)

19) targets cathepsin $B$ to 
91 endosomes/lysosomes (20) via mannose-6-phosphate receptor pathway. Propeptide exhibits an

92 essential role in the processing and maturation of cathepsin B. It acts as (a) a scaffold for

93 catalytic domain during protein folding, (b) involved in intracellular trafficking of cathepsin $B$ to

94 lysosome after N-terminal glycosylation and phosphorylation and (c) as a high-affinity reversible

95 inhibitor for the premature activation of zymogen. The crystal structure of procathepsin B [PDB

96 ID: 3PBH] has a propeptide region [ArP1 to LysP62], and main chain [Leu1 to Asp254] enzyme

97 residues (21). The main chain has two domains ( $\mathrm{R}$ and $\mathrm{L}$ domains) with active site residues

98 Cys29 and His199, located at the interdomain cleft. The propeptide siting in the active site cleft

99 is in reverse direction to that of the substrate, thus suggesting its role as an inhibitor. The

100 structure has an "occluding loop" [Ile105 to Pro126] which has an alternate conformation in

101 propeptide and in mature enzyme. The occluding loop is lifted above in procathepsin B, while it

102 is tightly packed in the active enzyme, thus exposing the active sites in Cathepsin B.

103 Procathepsin B is activated by other proteases like cathepsin $D$ and, also by autoactivation. The

104 potential intermolecular cleavage site identified in cathepsin B is CystP42-GlyP43.-At low pH,

105 acidic residues at propeptide surface destabilize-propeptide secondary structure, resulting in

106 distortion of hydrophilic and hydrophobic interaction with mature region of protein.

107 Subsequently, intermolecular cleavage takes place, and propeptide gets completely dissociated

108 from mature enzyme. Thus, autoactivation is a bimolecular process. Once CTSB gets activated,

109 it activates trypsinogen (22). Mutations affect different regions of Cathepsin B protein but how

110 these variants affect the function of Cathepsin $B$ is yet to be studied.

111 Since CTSB plays a cardinal role in premature trypsinogen activation, therefore in the present

112 study, we decided to analyze computationally the functional and structural effect of the missense

113 variants identified in the previous study (13), in order to determine the clinical significance of 
114 these mutations in TCP pathogenesis. We predicted the effect of these coding variants in the

115 propeptide region of cathepsin B, using various in silico algorithms. Also, we predicted that

116 variants present in 3'UTR region (noncoding) in cathepsin B are associated with miRNA binding

117 sites, and hence they could be significant. Evidential results from the structural and functional

118 analysis of SNVs in Cathepsin B have implicated the potential role of these variants in the

119 pathogenesis of tropical calcific pancreatitis. This study is the first attempt to structurally and 120 functionally characterize the variants found in human Cathepsin B protein screened in TCP 121 patients.

\section{Materials and Methods}

\section{Data curation}

124 The single nucleotide polymorphisms (SNPs) in CTSB gene associated with TCP was extracted 125 from an article published in 2006 (13). In this article, researchers have done direct exome 126 sequencing of CTSB gene, taking samples from 25 controls and 51 TCP patients, and further 127 replicating the sequencing in 130 controls and 89 TCP patients from the same cohort, in order to 128 ensure their results. In the current study, we have mapped the SNPs extracted from the literature on current human genome assembly, GRCh38.7. The mRNA accession number, NM_147782.2,

130 and protein accession number, NP_680092.1 of gene Cathepsin B (CTSB), was used in our 131 computational analysis. The current data about these SNVs were retrieved from human SNP 132 database, dbSNPbuild150. The workflow for the computational analysis performed to decipher 133 the significance of SNPs is depicted in Figure 1. 


\section{Sequence retrieval and Alignment}

136 The sequence of Cathepsin $B$ (CTSB) was retrieved from UniProt database- P07858

137 (CATB_HUMAN). The non-synonymous variants (L26V and S53G) were manually inserted in 138 the wild-type protein sequence for further analysis.

141 Non-synonymous SNP Analysis

142 The functional effect of mutations was predicted using the following algorithms: SIFT (Sorting 143 Intolerant from Tolerant) (23), PolyPhen-2 (Polymorphism Phenotyping v2) (24) and

144 PANTHER (25). SIFT predicts whether the non-synonymous coding mutation affects protein 145 function or not, based on sequence homology and physical properties of amino acids. SIFT 146 calculates median conservation value for each amino acid position and thus measures the 147 diversity of sequence. Finally, it gives the score which is the normalized probability of an amino 148 acid change. Score of less than 0.05 are deleterious substitutions. PolyPhen-2 predicts the impact 149 of substitution on structure and function of a protein, after annotating the substitutions and 150 finally building conservation profiles. The prediction algorithm of PolyPhen-2 calculates Naïve 151 Bayes posterior probability about the damaging effect of mutation and gives prediction 152 sensitivity scores also. PolyPhen-2 annotates the substitution as "Possibly damaging", "Probably 153 damaging" or "Benign", based on their scores. PANTHER predicts the functional effect of 154 coding mutation based on "evolutionary preservation" metric of a given substitution and 155 calculates preservation time- position-specific evolutionary preservation (PESP). Longer the 
156 PESP time, the more likely that substitution will have a deleterious effect. All these softwares:

157 SIFT, PolyPhen-2, and PANTHER, were based on evolutionary conservation-based algorithms.

158 Another tool, ProtParam (26) was used to calculate the hydropathicity or the GRAVY (grand

159 average of hydropathicity) score (27) of mutated procathepsin B sequences. Hydrogen bond

160 length and the rotational angles of main chain hydrogen bonds is a significant descriptor to study

161 the conformation and dynamics of a protein. Therefore, to calculate the altered hydrogen

162 bonding patterns in the mutated three-dimensional procathepsin B structures, Vadar v1.8

163 program (28) was used. This program calculates the H-bond distances in the main chain, side

164 chain and the bond angle between main chain residues.

165 Further, the stability of the mutated models was calculated by SDM (Site Directed Mutator)

166 server (29), I-Mutant suite (30) and mCSM (Mutation Cutoff Scanning Matrix Calculation) (31)

167 webservers. These algorithms calculate the difference in change in Gibbs free energy $(\Delta \Delta \mathrm{G})$.

168 SDM server is used to calculate the difference in thermal stability of wild-type protein structure

169 and mutated protein structure, using constrained environment specific substitution tables

170 (ESSTs). I-Mutant suite is a support vector machine-based algorithm which predicts the protein

171 stability upon mutation, by taking datasets from ProTherm (32) database. This server also

172 calculates the change in Gibbs free energy $(\Delta \Delta \mathrm{G})$ between wild-type and mutant protein

173 structures. mCSM server is used to predict the effect of mutations in proteins using graph-based

174 signatures. It predicts the effect of single point mutation on protein stability by extracting data

175 sets about various thermodynamic parameters from the ProTherm database and calculates change

176 in Gibbs free energy $(\Delta \Delta \mathrm{G})$ between wild-type and mutant protein structures. Altogether, these

177 three algorithms calculate $\Delta \Delta \mathrm{G}$ for a protein on mutation. 
178 The change in Gibbs free energy $(\Delta \Delta G)$ is as follows:

$179 \Delta \mathrm{G}=\Delta \mathrm{H}-\mathrm{T} \Delta \mathrm{S}$

$180 \Delta \Delta \mathrm{G}=\Delta \mathrm{G}_{\mathrm{W}}-\Delta \mathrm{G}_{\mathrm{M}}$

$181 \Delta \mathrm{G}=$ Change in Gibbs free energy of a system from unfavourable to favourable condition

$182 \Delta \mathrm{H}=$ Change in enthalpy of the system

$183 \Delta \mathrm{S}=$ Change in entropy of the system, $\mathrm{T}=$ Temperature of the system

$184 \Delta \Delta \mathrm{G}=$ Value of free energy stability change of a protein upon mutation

$185 \Delta \mathrm{G}_{\mathrm{W}}=$ Change in Gibbs free energy of the wild-type protein from unfavourable to favourable 186 conditions

$187 \Delta \mathrm{G}_{\mathrm{m}}=$ Change in Gibbs free energy of mutant from unfavourable to favourable conditions.

$188 \Delta \Delta \mathrm{G}>0=$ Increase protein stability upon mutation

$189 \Delta \Delta \mathrm{G}<0=$ Decrease protein stability upon mutation.

190 Additionally, we have also done multiple sequence alignment (MSA) of procathepsin B protein

191 using sequences from 8 model organisms along with Homo sapiens (NP_680092.1): Mus musculus

192 (NP_031824.1), Sus scrofa (NP_001090927.1), Macaca mulatta (NP_001181828.1), Rattus 193 norvegicus (NP_072119.2), Ovis aries (NP_001295516.1), Danio rerio (NP_998501.1), Bos

194 taurus (NP_776456.1). The MSA was performed using Clustal Omega program (33) 


\section{Homology modeling}

197 The two non-synonymous SNPs (L26V and S53G) were modelled to analyze the structural effect 198 of variants on protein. The position specific iterated blast program (PSI-BLAST) with protein 199 databank database (PDB) and default advanced settings, was used to find the template for 200 homology modelling. MODELLER 9.15 (34) was used to build mutated models of procathepsin

201 B. The best predicted models according to the lowest value of DOPE score (Discrete Optimized 202 Protein Energy), was used for further evaluation and analysis. The predicted 3D mutated models, 203 L26V and S53G, were evaluated for their quality by using Verify-3D (35) and ProSA (Protein 204 Structure Analysis) servers (36). Verify-3D examines the correctness of 3D-structures by 205 comparing the $3 \mathrm{D}$ structure to the $1 \mathrm{D}$ structure. If $3 \mathrm{D}-1 \mathrm{D}$ score of each amino acid is $\geq 1$, then 206 the model is correct. ProSA analysis the correctness of theoretical models by calculating the Z207 score of the input structure. ProSA considers C-alpha atoms of protein structure and calculates Z208 scores based on the similarity of crystal and NMR structures of the same size. If the Z-score for a 209 model is negative, then it is a model with minimum or no errors. After these models pass the respective thresholds, the Ramachandran plot was evaluated (37). TM-score (38) and root mean

211 square deviation (RMSD) values of mutant structures (L26V and S53G), were calculated with 212 respect to wild-type by using TM-Align web server (39).

\section{Molecular dynamics simulation}

214 The molecular dynamic simulation was performed with Gromacs-5.0 package (40) on the native 215 (PDB ID: 3PBH) and mutant structures (S53G and L26V). This computational investigation was 
216 done with a viewpoint to examine if these single nucleotide variants might lead to changes in

217 surface properties or distort the protein orientation. The protein molecule was solvated in a

218 dodecahedron box with SPC216 water molecules at $1.5 \AA$ marginal radiuses. The system was

219 made neutral by adding $7 \mathrm{Na}+$ (Sodium ions) because the initial charge of the system is -7.

220 Subsequently, the molecular system was subjected to steepest distance energy minimization until

221 reaching the criterion of $1000 \mathrm{~kJ} / \mathrm{mol}$ (the minimization is converged when maximum force is

222 less than $1000 \mathrm{~kJ} / \mathrm{mol}$ ) with OPLS-all atom force field (40). Berendson temperature coupling

223 method (41) was used to regulate the temperature inside the box at 300k. Isotropic pressure

224 coupling was performed using Parinello-Rahman method (42), and the pressure of the system

225 was maintained at 1 bar. LINCS algorithm (43) was used to treat bond lengths including H-

226 bonds. Van der Waals and Coulomb interactions were truncated at $1 \mathrm{~nm}$, and Particle Mesh

227 Ewald method (44) was used to compute electrostatic interaction. Finally, the simulation was

228 performed for $35 \mathrm{~ns}$. The structural deviations between native and mutated structures were

229 subjected to comparative analysis by computing RMSD (Root mean square deviation) and

230 RMSF (Root mean square fluctuation). The trajectories were analyzed, and finally, protein

231 compactness was studied by calculating the radius of gyration $(\mathrm{Rg})$. The secondary structure

232 analysis of wild-type and simulated mutant structures was also done using the do-dssp program 233 of Gromacs.

236 5'UTR and 3'UTR regions in a gene play a crucial role in regulating gene expression at the post-

237 translational level. UTRs regulate the exit of mRNAs from the nucleus, translation efficiency, 
238 sub-cellular localization and mRNA stability (45). The effect of SNPs in UTR regions was

239 analyzed using databases like1. miRdSNP (46), 2. PolymiRTS (47) and 3. miRNASNP (48).

240 Results

241 Data curation

242 The SNPs associated with TCP were extracted from literature and is tabulated in Table 1. The

243 SNPs were further categorized according to their type. There were total of 23 SNPs in CTSB

244 gene found to be associated with TCP. The non-coding region included 20 SNPs (1 deletion, 6 in

245 5'UTR, 2 in 3'UTR and 11 in introns). Coding region had 2 missense variants and 1 synonymous

246 variant (Table 1).

247 [Insert Table 1]

248

249 Sequence retrieval and Alignment

250 The protein sequence (NP_680092.1) was retrieved from the UniProt database, and the desired

251 variants were manually inserted in the sequence (Figure 2). The UniProt ID for the procathepsin

252 B sequence is P07858 (CATB_HUMAN). Mutant 1 (L26V) where residue L (Leucine) is

253 substituted with residue V (Valine). Mutant 2 (S53G) where residue S (Serine) is substituted

254 with residue G (Glycine).

255 [Insert Figure 2]

256 Homology modeling 
257 The 3D-structure of mutant (L26V and S53G) proteins was predicted after template searching by

258 PSI-BLAST. The protein sequence (NP_680092.1) of procathepsin B was used as a query, and

259 the resulting templates were then filtered. Finally, the X-ray crystal structure of human

260 procathepsin B (PDB ID: 3PBH) with $2.5 \AA$ resolution (Sequence identity: 100\% and Query

261 coverage: 93\%) was used as a template for homology modeling. The DOPE scores, TM-scores,

262 and RMSD of the predicted best models by Modeller 9.15 are shown in Table 2. The

263 stereochemical properties of the mutated procathepsin B structures were evaluated using the

264 Ramachandran plot from RAMPAGE. The plot defines the amino acids in favoured, allowed and

265 outlier regions in the mutated structures as well as in the wild-type Cathepsin B structures (Table

266 3). Verify-3D did structure validation of predicted models, and it was observed that $99.05 \%$

267 amino acids had average 3D-1D protein score in a 21 residue sliding window $>=0.2$ for L26V

268 mutated model and 97.16\% amino acids had average 3D-1D protein score in a 21 residue sliding

269 window $>=0.2$ in S53G mutated model. Additionally, the ProSA web server was also used to

270 evaluate the quality of predicted 3D mutated models. The Z-score (by ProSA webserver) of

271 L26V model was -7.32 and of S53G model was -7.47 , which were within the acceptable range of

272 X-ray and NMR studies. The interaction energy analyzed by ProSA tool was negative for

273 maximum residues in L26V, and S53G predicted models, in a sliding window of 10 and 40

274 respectively. Since the mutations were present in the propeptide region of procathepsin B, only

275 the mutated propeptide structures are shown in Figure 3 and Figure 4.

276 [Insert Table 2]

277 [Insert Table 3]

278 [Insert Figure 3] 
279

280

281

282

283

284

285

286

287

288

289

290

291

292

293

294

295

296

297

298

299

\section{[Insert Figure 4]}

\section{Non-Synonymous SNP Analysis}

The functional effect of the mutations predicted by using algorithms described in the "Methods" section are tabulated in Table 4. Analysis by SIFT and PANTHER suggests that S53G and L26V mutations can have damaging effects. The GRAVY scores of wild-type WT was -0.470 , and the mutants (L26V and S53G) was -0.469 for both. Thus, it could be concluded that there is no significant effect of mutations on hydropathicity of the protein. The comparative analysis of hydrogen bond lengths and the rotational angles between WT and mutants were calculated using Vadar v1.5 server, at 10 different regions of protein, playing imperative role in the functioning of procathepsin B. Remarkable differences in the H-bond lengths and Bond angles between WT and mutants (S53G and L26V) were observed as represented in Figure 5. Hence, it could be interpreted that these mutations alter the binding between residues in mutated structures.;

The change in Gibbs free energy $(\Delta \Delta \mathrm{G})$ of the mutated structures calculated by SDM server, IMutant 2.0, and mCSM webservers indicatedestabilization of mutated proteins (Table 5). Additionally, the results of MSA revealed that leucine at $26^{\text {th }}$ position and Serine at $53^{\text {rd }}$ position are conserved residues. Extrapolating these results indicate that any alteration in conserved residues will affect the structure and function of the protein (Figure 6). Altogether, L26V and S53G mutations were predicted to have a deleterious effect on the structure and function of the protein, through non-synonymous SNP analysis algorithms.

\section{[Insert Table 4]}

\section{[Insert Table 5]}


300

301

302

303

304

305

306

307

308

\section{[Insert Figure 5]}

\section{[Insert Figure 6]}

\section{Analysis of SNPs in UTR region.}

UTRs play an essential role in mRNA processing during post-translational mechanism. Hence, the SNPs in the UTR region can significantly affect the functionality of UTRs, provided they affect the miRNA binding sites. The 3'UTR region is essential for microRNA (miRNA) binding which can lead to degradation or transcriptional suppression of mRNA and thus, can further affect the downstream processing. The databases miRdbSNP, PolymiRTS, miRNASNP, were used to predict the significance of SNPs in the 3'UTR region (Table 6). The two SNPs present in 3'UTR region, rs 709821 and rs8898, were predicted to be present in miRNA binding sites and therefore are significant. The SNP, rs8898 was predicted to create a new miRNA site, while rs709821 disrupt a non-conserved miRNA site as predicted by PolymiRTS database. The two miRNAs targeting CTSB gene having both non-coding variants, hsa-miR-96 and hsa-miR-1271, are pancreas specific miRNAs, deciphered from the miRNet database (49).

\section{Molecular dynamics simulations.}

The comparative analysis of trajectories by calculating RMSD, RMSF and Radius of gyration after MD simulation of 35ns, for both native and mutants, was performed. Interestingly, it was observed from RMSD of backbone residues, that both mutated structures (S53G and L26V) were conspicuously deviated from the native structure (PDB ID: 3PBH). To infer effect of mutations on the dynamic behaviour of each residue, RMSF of $\mathrm{C} \alpha$-atoms was calculated. There was fluctuation observed in mutated protein structures as compared to the native structure. The 
321 protein compactness was determined by the radius of gyration ( $\mathrm{Rg}$ ). It was observed that $\mathrm{Rg}$ of

322 mutated structures were distinctly fluctuated as compared to native structure, throughout the

323 simulation (Figure 7). The secondary structure analysis of wild-type and simulated mutant

324 structures, by do-dssp program, implicated that mutations had caused deviation to the protein

325 (Figure 8).

326 [Insert Figure 7]

\section{7 [Insert Figure 8]}

\section{Discussion}

329 Tropical Calcific Pancreatitis (TCP) has distinct morphological characteristics with undefined 330 etiology. The propeptide region of procathepsin B (Arg1 -Lys 62) i.e. the N-terminal part inhibits

331 the activity of Cathepsin $B$ in the pancreas, thereby regulating its premature activation, also act

332 as a scaffold for protein folding and as a chaperone for endosome/lysosomal trafficking. L26V

333 and S53G are the two missense variants observed in the propeptide region of Cathepsin B protein

334 in TCP patients. The in-silico SNP analysis of the mutated protein sequences, resulted in

335 alteration of secondary structure, thereby predicting an adverse-folding effect on the protein. The

336 phenotypic effect of the mutations was calculated using sequence analysis algorithms, and it was

337 observed that at least two algorithms indicated a deleterious effect of these mutations on protein

338 functionality. The free energy $(\Delta \Delta \mathrm{G})$ calculations of mutated proteins structures, by various

339 algorithms, indicated that mutations are destabilizing. Further, the comparative analysis of H-

340 bond distances between mutated and native 3D-structure of procathepsin B provided a-unique

341 information about the structural characteristics of motifs around main chain H-bonds which are 
342 altered in mutant protein structures, thereby affecting the function of the protein (50).

343 Additionally, MD simulation of mutated and native protein structures indicated that the

344 mutations distinctly deviate the structural conformation of procathepsin $\mathrm{B}$, thereby having a 345 deleterious effect on-downstream signalling mechanism. Thus, the structural and functional 346 analysis of mutated procathepsin B predicts the significance of these mutations in the propeptide

347 region of Cathepsin B. Hence, we could extrapolate from these results (in silico analysis of the 348 mutated structures and sequences) that both mutations (L26V and S53G) have a deleterious 349 effect on structure and function of protein. These results will provide a lead towards designing 350 the experimental research strategy on the mutations involved in the pathogenesis of TCP to 351 understand the disease etiopathogenesis.

\section{Conclusion}

353 The dearth of information about the etiopathogenesis of tropical calcific pancreatitis was the 354 driving force for this study. The literature only has the information about the SNVs in cathepsin $355 B$ gene associated with TCP and lacks the crucial theories about the relative effects of these 356 SNVs in the pathogenesis of TCP. In this study, we predicted the structural and functional effect 357 of cathepsin B SNVs which were identified in TCP patients in previous studies. The predicted 358 deleterious effect of these SNVs is a lead towards developing biomarkers and therapeutics for 359 TCP. Further studies in this direction will help in defining the pathophysiology of TCP, which is 360 still a conundrum. 
363 AS is thankful to Shiv Nadar University for providing all the necessary support to perform this

364 study.

365

366

367

368

369

370

371

372

References:

373 1. Pandol SJ, Gorelick FS, Gerloff A, Lugea A. Alcohol abuse, endoplasmic reticulum 374 stress and pancreatitis. Dig Dis.2010;28:776-82.

375 2. Witt H, Bhatia E.Genetic aspects of tropical calcific pancreatitis. Rev Endocr Metab 376 Disord.2008; 9(3):213-26.

377 3. Levy P, Dominguez-Munoz E, Imrie C, Lohr M, Maisonneuve P. Epidemiology of 378 chronic pancreatitis: burden of the disease and consequences.United Eur Gastroenterol J.2014; $3792: 345-54$.

380 4. Kereszturi É, Szmola R, Kukor Z, Simon P, Ulrich Weiss F, Lerch MM, Sahin-Tóth M. 381 Hereditary pancreatitis caused by mutation-induced misfolding of human cationic trypsinogen: a 382 novel disease mechanism. Human mutation. 2009;30(4):575-82.

383 5. Zhang Y-F, Deng H-L, Fu J, Zhang Y, Wei J-Q. Pancreatitis in hand-foot-And-mouth 384 disease caused by enterovirus 71. World J Gastroenterol.2016; 22(6):2149-52.

385 6. Sarner M, Cotton PB. Classification of pancreatitis. Gut. 1984; 25:756-9.

386 7. Barman KK, Premalatha G, Mohan V.Tropical chronic pancreatitis.Postgrad Med 387 J.2003;79:606-15

388 8. Paliwal S, Bhaskar S, Chandak GR. Genetic and phenotypic heterogeneity in tropical 389 calcific pancreatitis. World J Gastroenterol. 2014; 20(46):17314-23. 
390

391

392

393

394

395

396

397

398

399

400

401

402

403

404

405

406

407

408

409

410

411

412

413

414

415

416

417 18. Pungerčar JR, Caglič D, Sajid M, Dolinar M, Vasiljeva O, Požgan U, Turk D, Bogyo M, 418 Turk V, Turk B. Autocatalytic processing of procathepsin B is triggered by proenzyme activity. 419 The FEBS journal. 2009;276(3):660-8.

420 19. Katunuma N. Posttranslational processing and modification of cathepsins and cystatins. J 421 Signal Transduct. 2010; 375345.

422 20. Ghosh P, Dahms NM, Kornfeld S. Mannose 6-phosphate receptors: new twists in the tale. 423 Nature reviews Molecular cell biology. 2003; 4(3):202-13.

9. Hassan Z, Mohan V, Ali L, Allotey R, Barakat K, Faruque MO, Deepa R, McDermott MF, Jackson AE, Cassell P, Curtis D. SPINK1 is a susceptibility gene for fibrocalculous pancreatic diabetes in subjects from the Indian subcontinent. The American Journal of Human Genetics. 2002 Oct 1;71(4):964-8.

10. Midha S, Khajuria R, Shastri S, Kabra M, Garg PK. Idiopathic chronic pancreatitis in India: phenotypic characterisation and strong genetic susceptibility due to SPINK1 and CFTR gene mutations. Gut. 2010 Jun 1;59(6):800-7.

11. Singh G, Bhat B, Jayadev MS, Madhusudhan C, Singh A. mutTCPdb: a comprehensive database for genomic variants of a tropical country neglected disease-tropical calcific pancreatitis. Database. 2018;2018.

12. Lerch MM, Halangk W. Human pancreatitis and the role of Cathepsin B. Gut. 2006; 55(9):1228-30.

13. Mahurkar S, Idris MM, Reddy DN, Bhaskar S, Rao GV, Thomas V, Singh L, Chandak GR. Association of cathepsin B gene polymorphisms with tropical calcific pancreatitis. Gut. 2006;55(9):1270-5.

14. Mahurkar S, Bhaskar S, Reddy DN, Prakash S, Rao GV, Singh SP, Thomas V, Chandak GR. TCF7L2 gene polymorphisms do not predict susceptibility to diabetes in tropical calcific pancreatitis but may interact with SPINK1 and CTSB mutations in predicting diabetes. BMC medical genetics. 2008;9(1):80.

15. Xiao Y, Yuan W, Yu B, Guo Y, Xu X, Wang X, Yu Y, Gong B, Xu C. Targeted gene nextgeneration sequencing in Chinese children with chronic pancreatitis and acute recurrent pancreatitis. The Journal of pediatrics. 2017;191:158-63.

16. Singh S, Choudhuri G, Agarwal S. Frequency of CFTR, SPINK1, and cathepsin B gene mutation in North Indian population: connections between genetics and clinical data. The Scientific World Journal. 2014;2014.

17. Olson O.C, Joyce J.A. Cysteine cathepsin proteases: regulators of cancer progression and therapeutic response. Nature Reviews Cancer. 2015; 15(12):712-29. 
424 21. Podobnik M, Kuhelj R, Turk V, Turk D. Crystal structure of the wild-type human 425 procathepsin B at $2.5 \AA$ resolution reveals the native active site of a papain-like cysteine protease 426 zymogen. Journal of molecular biology. 1997; 271(5):774-88.

427 22. Halangk W, Lerch MM, Brandt-Nedelev B, Roth W, Ruthenbuerger M, Reinheckel T, 428 Domschke W, Lippert H, Peters C, Deussing J. Role of cathepsin B in intracellular trypsinogen 429 activation and the onset of acute pancreatitis. The Journal of clinical investigation. 2000 430 ;106(6):773-81.

431 23. Ng PC, Henikoff S. SIFT: Predicting amino acid changes that affect protein function. Nucleic 432 acids research. 2003; 31(13):3812-4.

433 24. Adzhubei IA, Schmidt S, Peshkin L, Ramensky VE, Gerasimova A, Bork P, Kondrashov AS, 434 Sunyaev SR. A method and server for predicting damaging missense mutations. Nature methods. $435 \quad 2010 ; 7(4): 248$.

436 25. Mi H, Muruganujan A, Casagrande JT, Thomas PD. Large-scale gene function analysis with 437 the PANTHER classification system. Nature protocols. 2013; 8(8):1551-66.

438

439

440

441

442

443

444

445

446

447

448

449

450

451

452

453

454

455
26. Bendtsen JD, Jensen LJ, Blom N, Von Heijne G, Brunak S. Feature-based prediction of nonclassical and leaderless protein secretion. Protein Engineering Design and Selection. 2004;17(4):349-56.

27. Kyte J, Doolittle RF. A simple method for displaying the hydropathic character of a protein. Journal of molecular biology. 1982;157(1):105-32.

28. Willard L, Ranjan A, Zhang H, Monzavi H, Boyko RF, Sykes BD, Wishart DS. VADAR: a web server for quantitative evaluation of protein structure quality. Nucleic acids research. 2003;31(13):3316-9.

29. Worth CL, Preissner R, Blundell TL. SDM - a server for predicting effects of mutations on protein stability and malfunction. Nucleic acids research.2011.

30. Capriotti E, Fariselli P, Casadio R. I-Mutant2.0: predicting stability changes upon mutation from the protein sequence or structure. Nucleic acids research. 2005; 33 (Web Server issue): W306-W310.

31. Douglas E. V. Pires, David B. Ascher and Tom L.Blundell. mCSM: predicting the effects of mutations in proteins using graph-based signatures.Bioinformatics. 2014; 30(3): 335-342.

32. Bava KA, Gromiha MM, Uedaira H, Kitajima K, Sarai A. ProTherm, version 4.0: thermodynamic database for proteins and mutants. Nucleic acids research. 2004 Jan 1;32(suppl_1):D120-1. 
456 33. Sievers F, Wilm A, Dineen D, Gibson TJ, Karplus K, Li W, Lopez R, McWilliam H, 457 Remmert M, Söding J, Thompson JD. Fast, scalable generation of high-quality protein multiple 458 sequence alignments using Clustal Omega. Molecular systems biology. 2011;7(1):539.

459 34. Šali A, Blundell TL. Comparative protein modelling by satisfaction of spatial restraints. 460 Journal of molecular biology. 1993;234(3):779-815.

461 35. Eisenberg D, Lüthy R, Bowie JU. VERIFY3D: assessment of protein models with three462 dimensional profiles. Methods in enzymology. 1997; 277:396.

463

464

465

466

467

468

469

470

471

472

473

474

475

476

477

478

479

480

481

482

483

484

485

486

487

36. Wiederstein M, Sippl MJ. ProSA-web: interactive web service for the recognition of errors in three-dimensional structures of proteins. Nucleic acids research 35(suppl 2). 2007; W407-W10.

37. S.C. Lovell, I.W. Davis, W.B. Arendall III, P.I.W. de Bakker, J.M. Word, M.G.Prisant, J.S. Richardson and D.C. Richardson. Structure validation by Calpha geometry: phi,psi and Cbeta deviation. Proteins: Structure, Function \& Genetics. 2002; 50: 437-450.

38. Zhang Y, Skolnick J. TM-align: a protein structure alignment algorithm based on the TMscore. Nucleic acids research. 2005; 33(7):2302-9.

39. H.J.C.Berendsen, D.van der Spoel,R.van Drunen. GROMACS: A message-passing parallel molecular dynamics implementation.Computer Physics Communications. 1995; 91:43-56.

40. Kutzner C, Páll S, Fechner M, Esztermann A, de Groot BL, Grubmüller H. Best bang for your buck: GPU nodes for GROMACS biomolecular simulations. Journal of computational chemistry. 2015;36(26):1990-2008.

41. Berendsen HJC, Postma JPM, van Gunsteren WF, DiNola A, Haak JR. Molecular dynamics with coupling to an external bath. J Chem Phys. 1984;81:3684-90.

42. Martoňák R, Laio A, Parrinello M. Predicting crystal structures: the Parrinello-Rahman method revisited. Physical review letters. 2003;90(7):075503.

43. Hess B, Bekker H, Berendsen HJ, Fraaije JG. LINCS: a linear constraint solver for molecular simulations. Journal of computational chemistry. 1997;18(12):1463-72.

44. Darden T, York D, Pedersen L. Particle mesh Ewald: an $\mathrm{N} \square \log (\mathrm{N})$ method for Ewald sums in large systems. J Chem Phys 1993;98:10089-92.

45. Flavio Mignone, Carmela Gissi, Sabino Liuni and Graziano Pesole. Untranslated regions of mRNAs.Genome Biology. 2002; 3(3).

46. Andrew E. Bruno, Li Li, James L. Kalabus, Yuzhuo Pan, Aiming Yu, Zihua Hu miRdSNP: a database of disease-associated SNPs and microRNA target sites on 3'UTRs of human genes. BMC Genomics.2012; 13(1):44. 
488 47. Bhattacharya A, Ziebarth JD, Cui Y. PolymiRTS Database 3.0: linking polymorphisms in 489 microRNAs and their target sites with human diseases and biological pathways. Nucleic Acids 490 Res.2014; 42(D1):D86-D91.

491 48. Jing Gong, Yin Tong, Hong-Mei Zhang, An-Yuan Guo. miRNASNP: a database of miRNA 492 related SNPs and their effects on miRNA function. BMC Bioinformatics.2012; 13:A2.

493 49. Fan Y, Siklenka K, Arora SK, Ribeiro P, Kimmins S, Xia J. miRNet-dissecting miRNA494 target interactions and functional associations through network-based visual analysis. Nucleic 495 acids research. 2016 Apr 21;44(W1):W135-41.

496 50. Penner RC, Andersen ES, Jensen JL, Kantcheva AK, Bublitz M, Nissen P, Rasmussen AM, 497 Svane KL, Hammer B, Rezazadegan R, Nielsen NC. Hydrogen bond rotations as a uniform 498 structural tool for analyzing protein architecture. Nature communications. 2014; 17;5. 
Figure 1

Workflow to identify the potential effect of SNPs

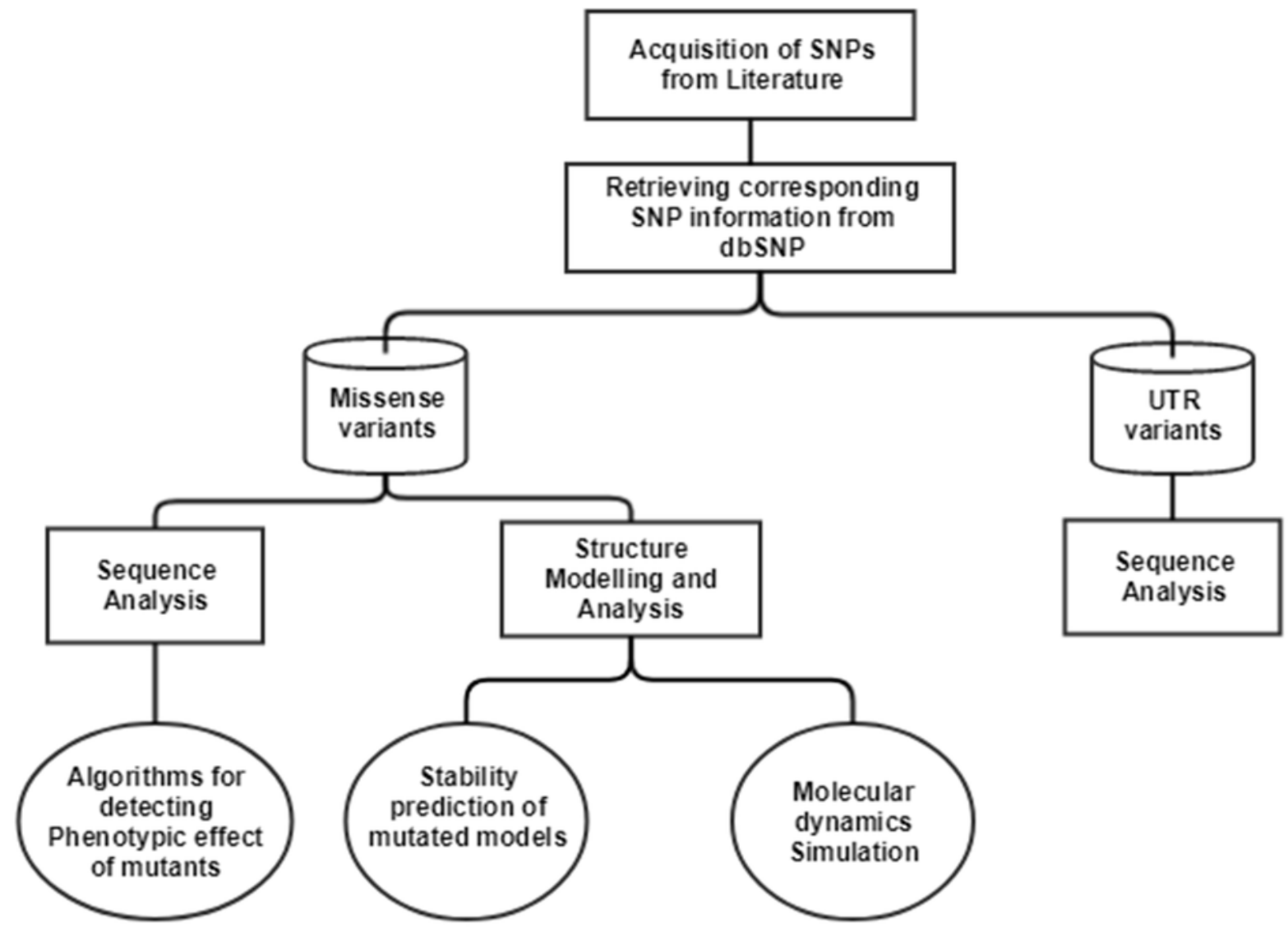




\section{Figure 2}

Fasta alignment of procathepsin B protein retrieved from Uniprot database

(A) Fasta sequence of wild-type procathepsin B (NP_680092.1, Isoform 1) retrieved from Uniprot database (ID : P07858). The wild type amino acids which were mutated in TCP patients, are highlighted in red

(A). $>$ sp $\mid$ P07858 $\mid$ CATB_HUMAN Cathepsin B OS=Homo sapiens GN=CT SB PE=1 SV=3 Native MWQLWA SL CCLLVLANARSRPSFHPLSDELVNYVNKRNTTWQAGHNFYNVDMSY LKRLCGTFL GGPKPPQRVMFTEDL KLPASFDAREQWPQCPTIKEIRDQGSCGSCWAF GAVEAISDRICIHT NAHV SVEVSAEDLLT CCGSMCGDGCNGGYPAEAWNFWTRKGL VSGGLYESHV GCRPYSIPPCEHHVNGSRPPCT GEGDT PKCSKICEPGYS PT YKQDKHY GYNSYSVSNSEKDIMA EIY KNGPVEGAFSVYSDFLLY KSGVYQHVTGEMMGGHAIR ILGWGVENGTPYWLVANSWNTDWGDNGFF KILRGQDHCGIESEVV AGIPRTDQYW EKI 


\section{Figure 3}

Mutated (orange color) and wild-type (black color) propeptide models are shown

The mutation, L26V is shown in sticks, which is equivalent to LEU9P-VAL10 in PDB files.

Visualization and numbering is done using PyMOL tool. Note: Numbering of amino acids in wild type PDB (3PBH) file differ to that of mutated models because propeptide and peptide regions are numbered separately in the published wild-type PDB file. 


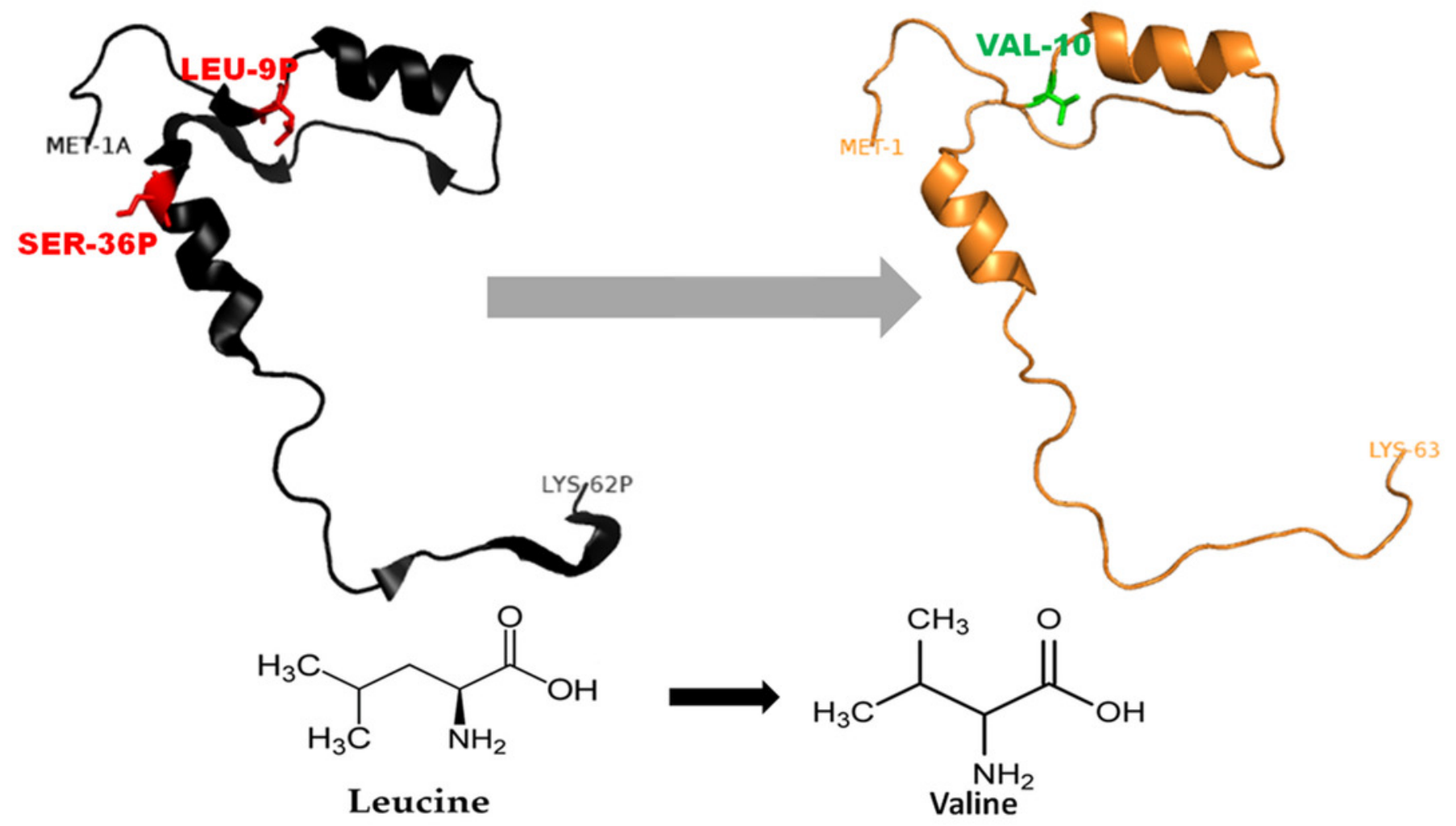


Figure 4

Mutated (Blue color) and wild-type (black color) propeptide models are shown.

The mutation, S53G is shown insticks as SER36-GLY37 in PDB files. Visualization and numbering is done using PyMOL tool. Note: Numbering of amino acids in wild type PDB (3PBH) file differ to that of mutated models because propeptide and peptide regions are numbered separately in the published wild-type PDB file. 


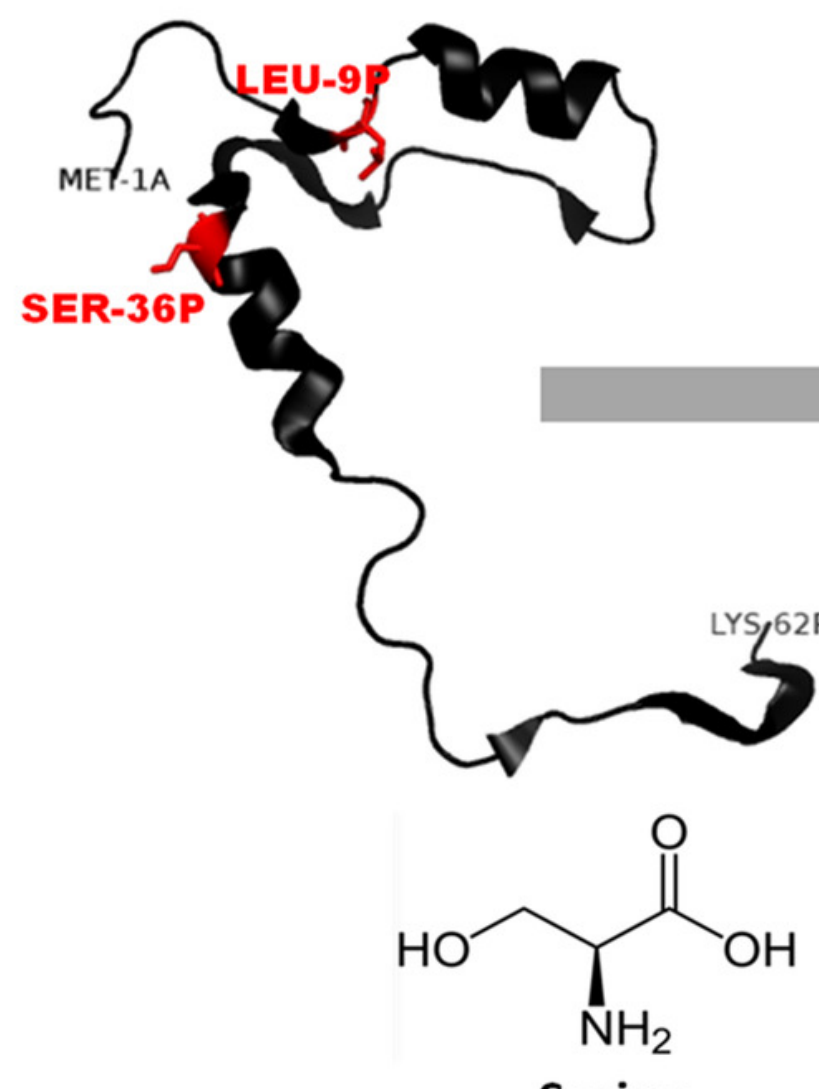

Serine

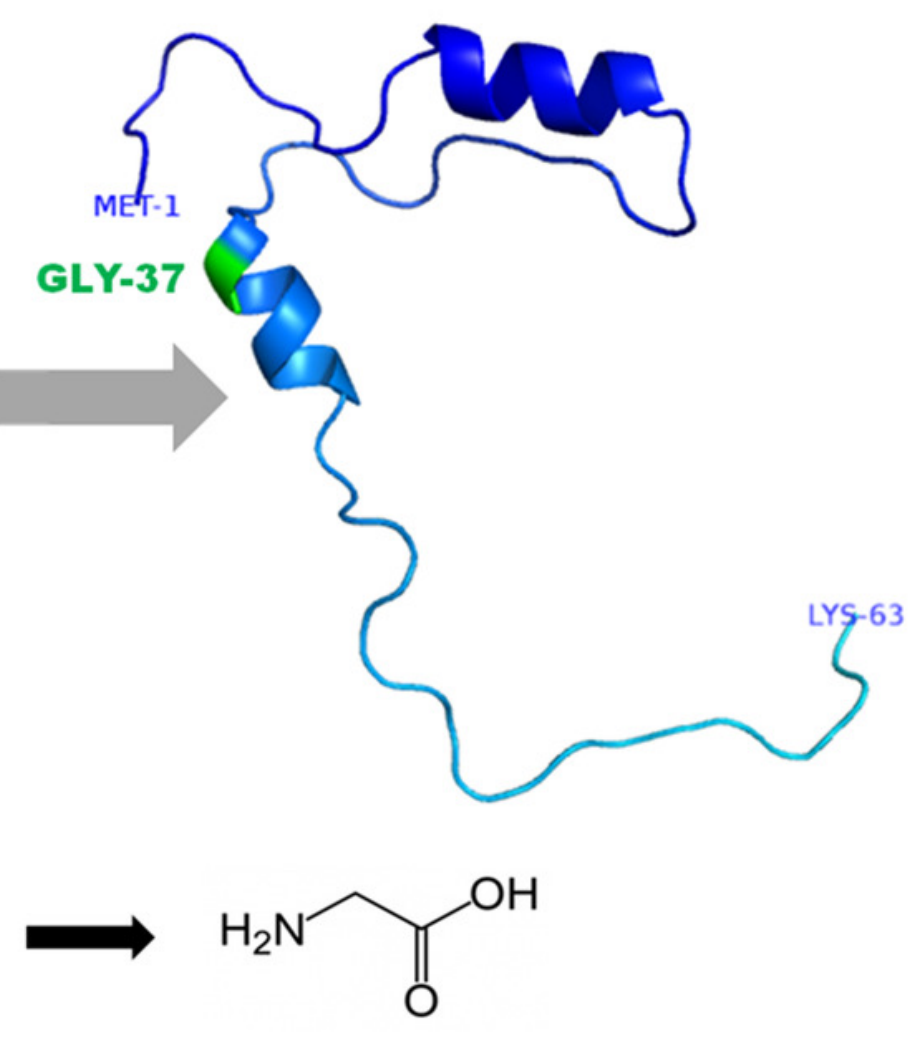

Glycine 


\section{Figure 5}

The comparative analysis of $\mathrm{H}$-bond length between wild-type (WT) procathepsin $B$ protein (PDB ID: 3PBH) and

A. Mutated structure (L26V) having a mutation at $26^{\text {th }}$ amino acid (Leucine to Valine) in propeptide region B. Mutated structure (S53G) having a mutation at $53^{\text {rd }}$ position (Serine to Glycine) in propeptide region. The colour key ranges from $1.5 \AA$ to $3.5 \AA$ with red as strong $\mathrm{H}$ bonding and blue as weak $\mathrm{H}$-bonding. Wide spaces indicate the absence of $\mathrm{H}$-bonds at that position. 

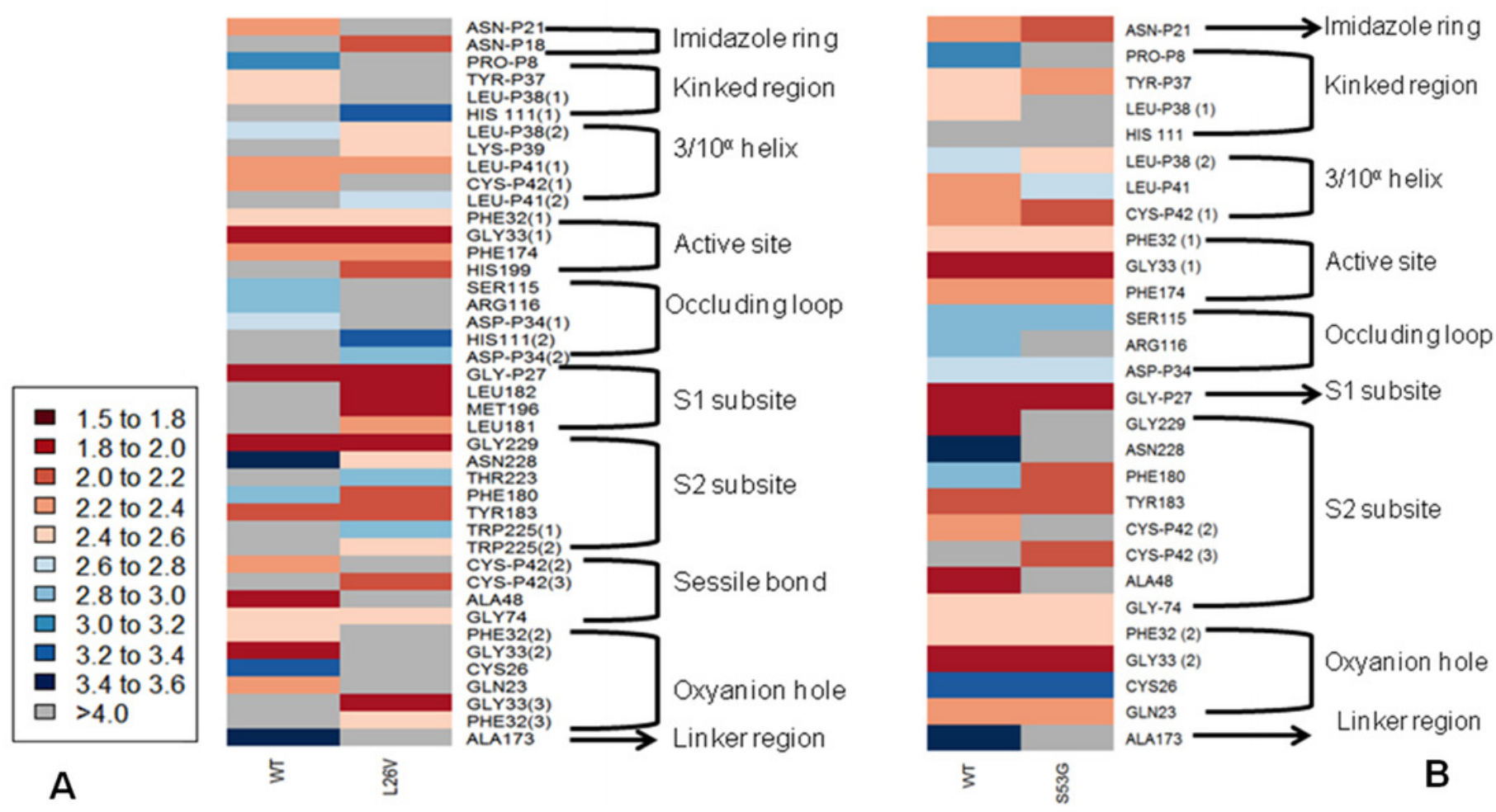


\section{Figure 6}

\section{Multiple sequence allignment}

\section{(A). MSA of wild type procathepsin B protein (NP_680092.1). (B.) MSA of mutated protein}

sequence with mutation S53G. (C) MSA of mutated protein sequence with mutation L26V.

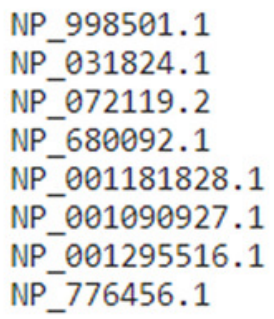

C

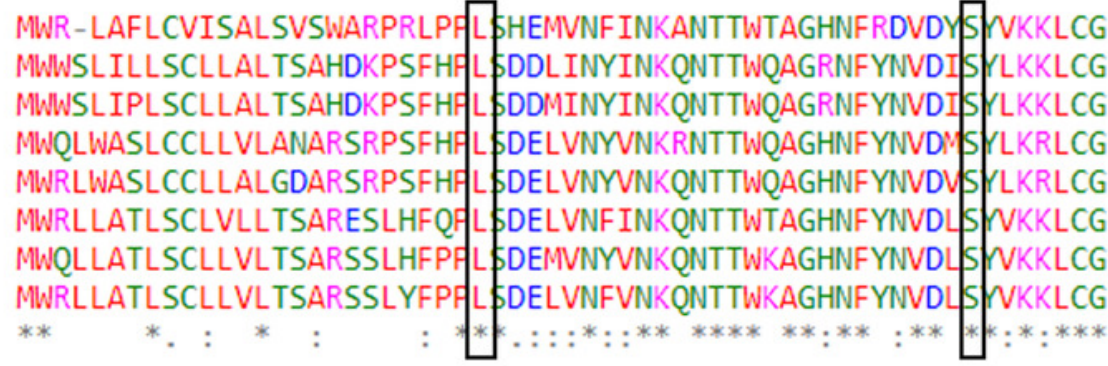

MWR-LAFLCVISALSVSWARPRLPPLSHEMVNFINKANTTWTAGHNFRDVDYFWKKLCG MWWSLILLSCLLALTSAHDKPSFHPLSDDL INYINKONTTWQAGRNFYNVDI $\$$ LKKLCG MWWSLIPLSCLLALTSAHDKPSFHPLSDDMINYINKQNTTWQAGRNFYNVDI $\$$ LKKLCG MWQLWASLCCLLVLANARSRPSFHPLSDELVNYVNKRNTTWQAGHNFYNVDM 5 SLKRLCG MWRLWASLCCLLALGDARSRPSFHPLSDELVNYVNKQNTTWQAGHNFYNVDVE MWRLLATLSCLVLLTSARESLHFQPLSDELVNFINKONTTWTAGHNFYNVDL $\$$ VKKLCG MWQLLATLSCLLVLTSARSSLHFPPLSDEMVNYVNKONTTWKAGHNFYNVDLE VVKKLCG MWRLLATLSCLLVLTSARSSLYFPPLSDELVNFVNKONTTWKAGHNFYNVDLF VVKKLCG

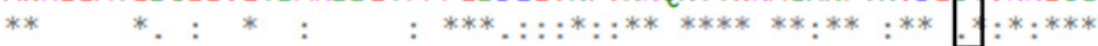

MWR - LAFLCVISALSVSWARPRLPP DHEMVNFINKANTTWTAGHNFRDVDYSYVKKLCG MWWSL ILLSCLLALTSAHDKPSFHP L \$DDL INYINKQNTTWQAGRNFYNVDISYLKKLCG MWWSLIPLSCLLALTSAHDKPSFHP L \$DDINYINKQNTTWQAGRNFYNVDISYLKKLCG MWQLWASLCCLLVLANARSRPSFHPV \$DELVNYVNKRNTTWQAGHNFYNVDMSYLKRLCG MWRLWASLCCLLALGDARSRPSFHPL \$DELVNYVNKQNTTWQAGHNFYNVDVSYLKRLCG MWRLLATLSCLVLLTSARESLHFQP L DELVNFINKQNTTWTAGHNFYNVDLSYVKKLCG MWQLLATLSCLLVLTSARSSLHFPP L \$DEMVNYVNKQNTTWKAGHNFYNVDLSYVKKLCG MWRLLATLSCLLVLTSARSSLYFPP L \$DLVNFVNKQNTTWKAGHNFYNVDLSYVKKLCG *** * : * : : : : : : : : *: : ********:**:****:*:*** 
Figure 7

MD Simulation

(A) Root mean square deviation (RMSD)-Backbone: Comparative analysis of RMSDbackbone between Wild-type and both mutated structures.(B). Root mean square

Fluctuation (RMSF)-C-alpha: Comparative analysis RMSF between mutated (s53g and I26v) and native protein structures. (C). Radius of gyration ( $\mathbf{R g})$ : Comparative analysis of $\mathbf{R g}$ between wild-type protein (s53g, 126v) and native protein structures. 


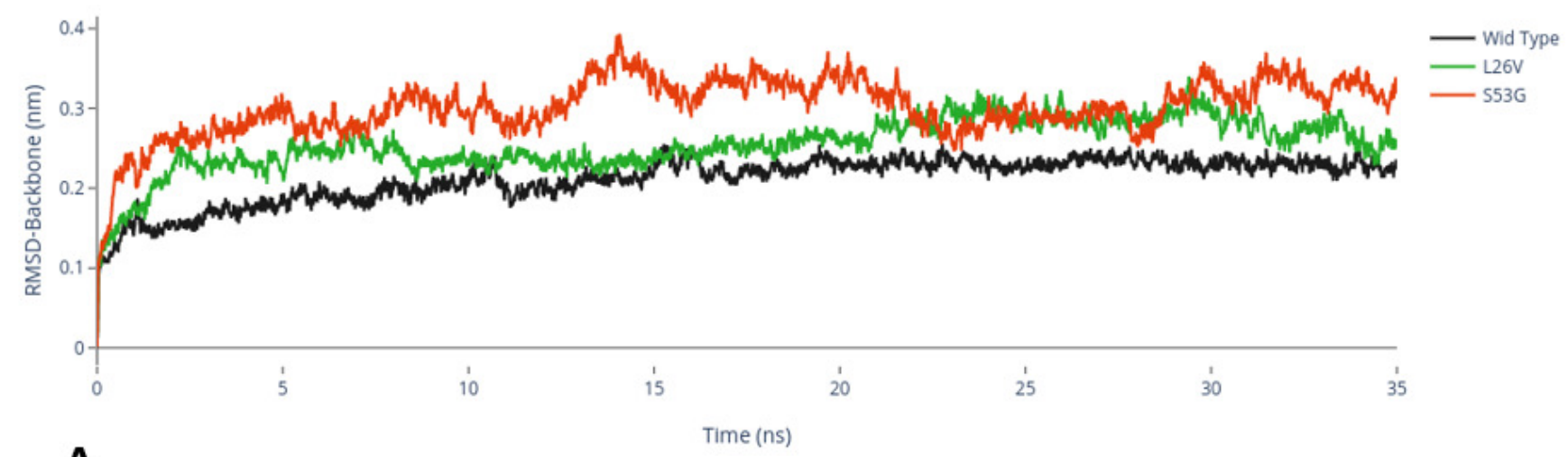

A
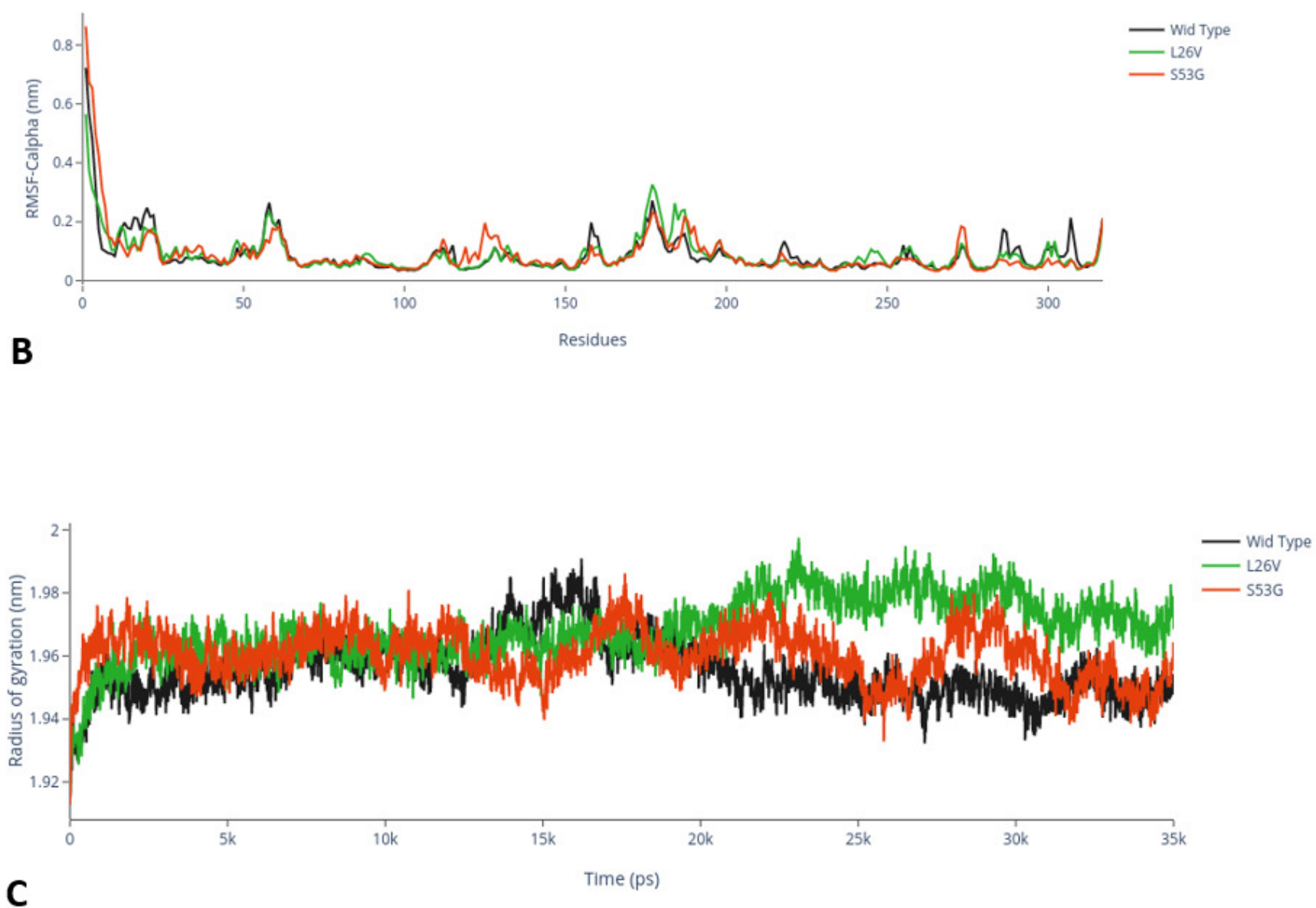
Figure 8

Secondary structure analysis:

The graphs depicts the secondary structure analysis using do-dssp program of (A) Wild type protein structure - 3PBH (B). Mutated structure 1: L26V and (C) Mutated protein structure 2: S53G 


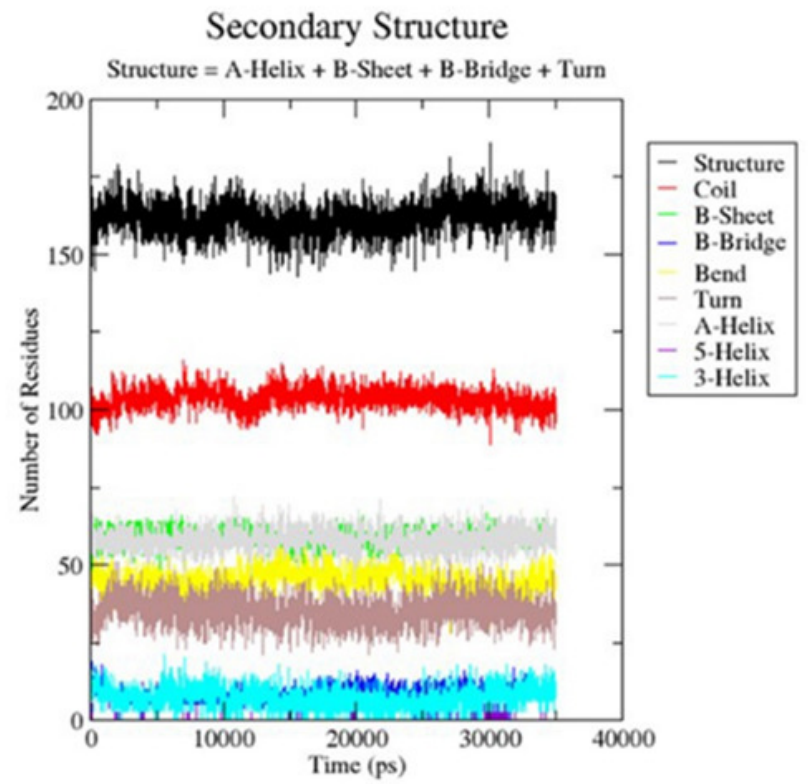

A 3PBH-WildType protein

Secondary Structure

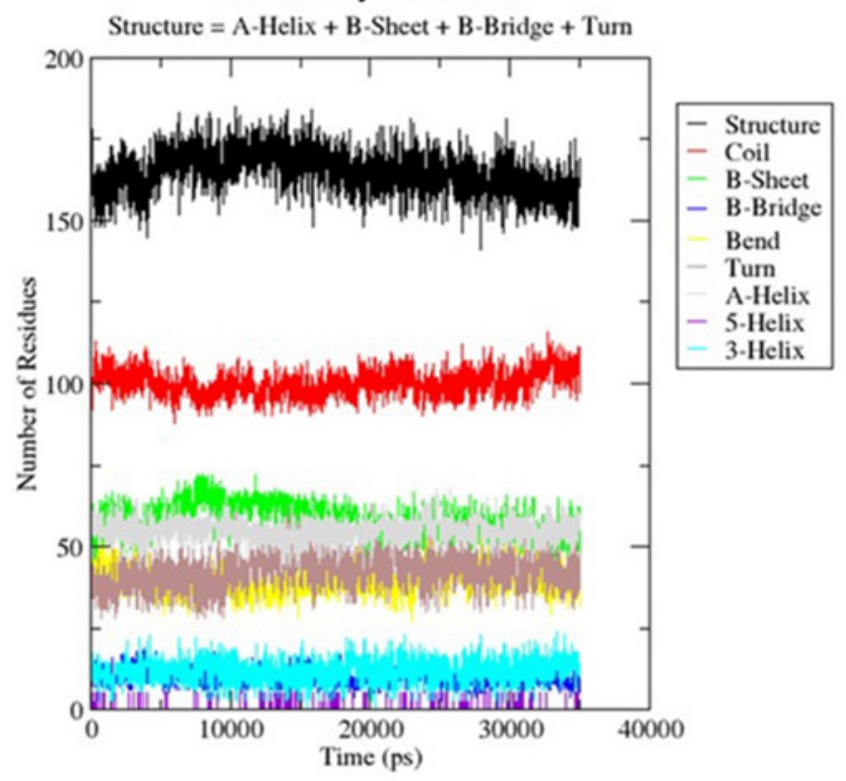

B

L26V

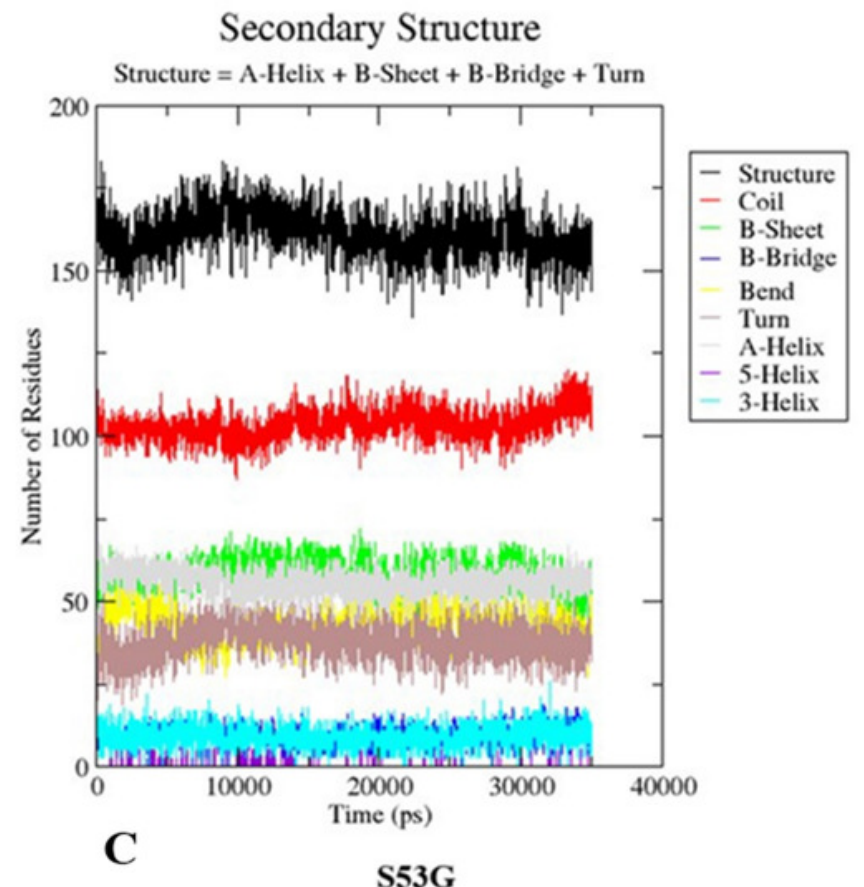




\section{Table $\mathbf{1}$ (on next page)}

The Single Nucleotide Polymorphisms in cathepsin B protein mined from literature (PMID: 16492714).

The SNP information is with respect to Ref Seq sequence ID: NT_077531.5 and dbSNP Build 150. 


\begin{tabular}{|c|c|c|c|c|c|c|}
\hline Ref rsID/ss ID & Position & Type & $\begin{array}{l}\text { CDS } \\
\text { position(relative } \\
\text { to CDS start) }\end{array}$ & $\begin{array}{l}\text { CDS } \\
\text { Allele } \\
\text { change }\end{array}$ & $\begin{array}{l}\text { Protein } \\
\text { Position }\end{array}$ & $\begin{array}{l}\text { Residue } \\
\text { change }\end{array}$ \\
\hline - & Exon 1(5'UTR) & Non coding & 14609 & $\mathrm{C}>\mathrm{A}$ & - & - \\
\hline- & Intron1(5'UTR) & Non coding & 14520 & $\mathrm{G}>\mathrm{C}$ & - & - \\
\hline - & Intron1(5'UTR) & Non coding & 14453 & $\mathrm{G}>\mathrm{A}$ & - & - \\
\hline rs1293311 & Intron1(5'UTR) & Non coding & 14425 & $\mathrm{C}>\mathrm{A}$ & - & - \\
\hline rs2645415 & Intron1(5'UTR) & Non coding & 11083 & $\mathrm{~T}>\mathrm{C}$ & - & - \\
\hline- & Exon 2(5'UTR) & Non coding & 10927 & $\mathrm{C}>\mathrm{G}$ & - & - \\
\hline rs4292649(rs12338) & Exon 3 & $\begin{array}{l}\text { Non-synonymous } \\
\text { coding(Missense) }\end{array}$ & 76 & $\mathrm{C}>\mathrm{G}$ & 26 & $\begin{array}{l}\mathrm{L}>\mathrm{V} \\
(\mathrm{Leu}>\mathrm{Val})\end{array}$ \\
\hline rs1293293(rs1122182) & Intron 3 & Non-coding & 335 & $\mathrm{~A}>\mathrm{T}$ & - & - \\
\hline- & Intron 3 & Non-coding & 394 & $\mathrm{G}>\mathrm{A}$ & - & - \\
\hline rs1293292 & Intron 3 & Non-coding & 595 & $\mathrm{C}>\mathrm{T}$ & - & - \\
\hline rs1293291 & Intron 3 & Non-coding & 663 & $\mathrm{~T}>\mathrm{C}$ & - & - \\
\hline rs1803250 & Exon 4 & $\begin{array}{l}\text { Non-synonymous } \\
\text { coding(Missense) }\end{array}$ & $790^{*}$ & $\mathrm{~A}>\mathrm{G}$ & 53 & $\begin{array}{l}\mathrm{S}>\mathrm{G} \\
(\mathrm{Ser}>\text { Gly) }\end{array}$ \\
\hline rs2272766 & Intron 5 & Non-coding & 2609 & $\mathrm{C}>\mathrm{T}$ & - & \\
\hline rs 13332 & Exon 6 & $\begin{array}{l}\text { Synonymous } \\
\text { coding }\end{array}$ & $4383^{*}$ & $\mathrm{~A}>\mathrm{C}$ & 140 & $\begin{array}{l}\mathrm{T}>\mathrm{T} \\
(\mathrm{Thr}>\mathrm{Thr})\end{array}$ \\
\hline - & Intron 6 & Non-coding & 4451 & $\mathrm{G}>\mathrm{C}$ & - & - \\
\hline rs1736090 & Intron 6 & Non-coding & 4735 & $\mathrm{~A}>\mathrm{G}$ & - & - \\
\hline rs1692819 & Intron 7 & Non-coding & 5516 & $\mathrm{C}>\mathrm{T}$ & - & - \\
\hline rs2294139 & Intron 7 & Non-coding & 5522 & $\mathrm{C}>\mathrm{A}$ & - & - \\
\hline rs3215434 & Intron 7 & $\begin{array}{l}\text { Non- } \\
\text { coding(Deletion) }\end{array}$ & $5581-5582$ & & - & - \\
\hline- & Intron 7 & Non-coding & 5622 & $\mathrm{C}>\mathrm{G}$ & - & - \\
\hline rs2294138 & Intron 8 & Non-coding & 5825 & $\mathrm{G}>\mathrm{A}$ & - & - \\
\hline rs709821 & $\begin{array}{l}\text { Exon } \\
11\left(3^{\prime} \text { UTR }\right)\end{array}$ & Non-coding & $8370^{*}$ & $\mathrm{C}>\mathrm{G}$ & - & - \\
\hline rs8898 & $\begin{array}{l}\text { Exon } \\
11\left(3^{\prime} \text { UTR }\right)\end{array}$ & Non-coding & $8422 *$ & $\mathrm{~A}>\mathrm{G}$ & - & - \\
\hline
\end{tabular}




\section{Table 2 (on next page)}

Quality assessment scores after modelling protein structures:

DOPE scores after homology modelling by Modeller 9.15 of mutants (L26V and S53G) and the structure alignment scores (TM-score and RMSD) of the CTSB mutant models with wild-type, 3PBH structure. 
1

2

3

4

\begin{tabular}{lllll}
\hline Predicted & \multicolumn{1}{c}{ DOPE Score } & & TM-score & RMSD \\
\cline { 2 - 2 } mutant model & Modeller 9.15 & & \multicolumn{2}{c}{ TM-Align } \\
& & & \\
\hline L26V & -34105.02344 & & 0.99941 & 0.16 \\
S53G & $-34285-52344$ & & 0.99973 & 0.17 \\
\hline
\end{tabular}




\section{Table 3 (on next page)}

The Ramachandran plot analysis of mutated models:

The table enlists the analysis from Ramachandran plot for each of the mutated protein structures (L26V and S53G) 
1

2

3

4

5

6

\begin{tabular}{llll}
\hline Models & Favoured & Allowed & Outlier \\
& region & region & region \\
\hline L26V & & & \\
S53G & $92.7 \%$ & $5.1 \%$ & $2.2 \%$ \\
\hline
\end{tabular}

7 


\section{Table 4 (on next page)}

Prediction of functional effect of mutations by using different algorithms:

The table enlists the scores from SIFT, Polyphen-2 and PANTHER for each mutated protein sequence: L26V and S53G. 
1

2

3

4

5

6

7

\begin{tabular}{|l|l|l|l|l|l|l|l|l|}
\hline & & & \multicolumn{2}{|c|}{ SIFT } & \multicolumn{2}{c|}{ Polyphen 2 } & \multicolumn{2}{c|}{ PANTHER } \\
\hline rsID & Allele & AA & Score & Prediction & Score & Prediction & Score & Prediction \\
& change & change & & & & & & \\
\hline rs4292649(rs12338) & C $>$ G & L26V & 0 & Affect protein & 0.01 & Benign & 1629 & Probably \\
& & & & & function & & & damaging \\
\hline rs1803250 & A $>$ G & S53G & 0.02 & Affect protein & 0.06 & Benign & 750 & Probably \\
& & & & function & & & & damaging \\
\hline
\end{tabular}




\section{Table 5 (on next page)}

Prediction of protein (procathepsin $B$ ) stability upon mutation:

The table enlists the change in Gibbs free energy $(\Delta \Delta \mathrm{G})$ in $\mathrm{kcal} / \mathrm{mol} . \Delta \Delta \mathrm{G}>0$ indicates stabilization while $\Delta \Delta G<0$ indicates destabilization. 
1

2

3

4

5

6

7

8

9

10

\begin{tabular}{|l|l|l|l|}
\hline Algorithm & S53G & L26V & Effect \\
\hline SDM & -.174 & -0.94 & Destabilizing \\
\hline I-Mutant 2.0 & -1.48 & -1.93 & Destabilizing \\
\hline mCSM & -1.063 & -1.638 & Destabilizing \\
\hline
\end{tabular}


Table 6(on next page)

The SNPs in 3'UTR region of CTSB protein:

The table enlists the predicted miRNAs targeting the CTSB gene sequence having 3'UTR SNVs. 


\begin{tabular}{|c|c|c|c|c|c|}
\hline rsID & Region & $\begin{array}{l}\text { Allele } \\
\text { change }\end{array}$ & miRdSNP & PolymiRTS & miRNASNP \\
\hline \multirow{12}{*}{ rs709821 } & \multirow{12}{*}{ UTR-3 } & \multirow{12}{*}{$\mathrm{C}>\mathrm{G}$} & hsa-miR-186 & - & - \\
\hline & & & hsa-miR-339-5p & - & - \\
\hline & & & hsa-miR-7 & - & - \\
\hline & & & hsa-miR-214 & - & - \\
\hline & & & hsa-miR-431 & - & - \\
\hline & & & hsa-miR-186 & - & - \\
\hline & & & hsa-miR-320a & - & - \\
\hline & & & hsa-miR-320d & - & - \\
\hline & & & hsa-miR-320c & - & - \\
\hline & & & hsa-miR-320b & - & - \\
\hline & & & hsa-miR-96 & - & - \\
\hline & & & hsa-miR-1271 & - & - \\
\hline \multirow{10}{*}{ rs8898 } & \multirow{10}{*}{ UTR-3 } & \multirow{10}{*}{$\mathrm{A}>\mathrm{G}$} & hsa-miR-339-5p & hsa-miR-10a-5p & $\begin{array}{l}\text { hsa-miR-10a- } \\
5 \mathrm{p}\end{array}$ \\
\hline & & & hsa-miR-186 & hsa-miR-10b-5p & $\begin{array}{l}\text { hsa-miR-10b- } \\
5 p\end{array}$ \\
\hline & & & hsa-miR-7 & hsa-miR-339-5p & $\begin{array}{l}\text { hsa-miR-339- } \\
5 p\end{array}$ \\
\hline & & & hsa-miR-214 & hsa-miR-4421 & \\
\hline & & & hsa-miR-431 & $\begin{array}{l}\text { hsa-miR-5699- } \\
3 \mathrm{p}\end{array}$ & \\
\hline & & & hsa-miR-186 & $\begin{array}{l}\text { hsa-miR-6747- } \\
3 \mathrm{p}\end{array}$ & \\
\hline & & & hsa-miR-320a & $\begin{array}{l}\text { hsa-miR-6752- } \\
3 \mathrm{p}\end{array}$ & \\
\hline & & & hsa-miR-320d & & \\
\hline & & & hsa-miR-320c & & \\
\hline & & & hsa-miR-320b & & \\
\hline
\end{tabular}




\begin{tabular}{|l|l|l|l|l|l|}
\hline & & & hsa-miR-96 & & \\
\cline { 3 - 5 } & & & hsa-miR-1271 & & \\
\hline
\end{tabular}

1 